\title{
Automatic Argumentation Extraction
}

\author{
Alan Sergeant \\ SAP UK Ltd, The Concourse, Queen's Road, Belfast, BT3 9DT
}

\begin{abstract}
This extended abstract outlines the area of automatic argumentation extraction. The state of the art is discussed, and how it has influenced the proposed direction of this work. This research aims to provide decision support by automatically extracting argumentation from natural language, enabling a decision maker to follow more closely the reasoning process, to examine premises and counter-arguments, and to reach better informed decisions.
\end{abstract}

Keywords: Argumentation, Argument Extraction, Information Extraction.

\section{Problem Overview}

Automatic Argumentation Extraction (AAE) is a relatively new research area [1, and work carried out to date is still regarded as experimental[2]. Argumentation can be defined as the process by which arguments are constructed and handled [3], with four main tasks undertaken: identification, analysis, evaluation and invention 4. Identification is the task of determining the conclusion, premises and scheme of an argument from natural discourse, and is the task with which this work is concerned with automating.

Motivation for this work comes from the question "Was the right decision made? Was it well founded?" For every decision made, one might be asked to justify, explain or defend how it was arrived at [5]. An antagonist can probe the reasoning process which led to the conclusion by asking for clarification or justification. Therefore, it can be said that arguments are constructed to express the reasoning process taken to reach a conclusion, with a view to persuading hearers that the conclusion is valid and the reasoning behind it well grounded 6 .

What is an argument? The building blocks of every argument are propositions: a statement or assertion that expresses a judgement or opinion [7. An argument consists of two or more propositions 38 , one proposition functions as the claim (also known as the conclusion), and a set of one or more propositions serve as supports (also known as premises). The relationship between the propositions (premises and conclusions) is important. An argument is not simply a collection of propositions, it has a structure, which plays a key role in determining the presence or absence of an argument [7].

One useful approach to viewing arguments and their structure is that of argument diagramming. Argument diagramming enables the conclusions and related premises to be identified, and the relationships between them expressed in a tree

P. Cimiano et al. (Eds.): ESWC 2013, LNCS 7882, pp. 656-660, 2013.

(C) Springer-Verlag Berlin Heidelberg 2013 
structure 9. It provides an overview of how well supported or attacked a conclusion or premise is. This overview can be used to inform argument-based decision making. The argument diagram is a representation of the reasoning process, and serves as a basis for reflection on how the conclusion was reached. It also enables an antagonist to target certain areas in the reasoning process for further examination. If a conclusion is well supported, or even if it has been attacked and successfully defended, it provides good ground on which to make a decision, as the reasoning process by which it was reached is demonstrated to be valid.

Automatic argumentation extraction incorporates the understanding of construction, handling, and visual representation of arguments and aims to support decision making. Given the new ways in which we communicate (newspapers, Facebook, Twitter, review sites etc.), often statements or assertions are made without explicit justification for the opinion, belief or conclusion. Without this, how can a reader reasonably decide to agree with a post or meaningfully assess whether a product is suitable for purchase?

This research will begin by finding means of automatically identifying argumentative propositions (premises or conclusions). However at this first stage no attention to type (whether a proposition is premise or conclusion) is considered. It has been shown that by filtering out propositions that have no role to play in an argument, a more accurate classification of type can be achieved[1]. Finally, once the propositions have been classified by their type, work will move towards identifying the relationship (support or attack) between the premises and conclusions, forming an argument diagram.

\section{Related Work}

While so far there has been little work in the area of AAE 112], several related areas have been the focus of research: text zoning 10, RST (rhetorical structure theory) 11, argumentation schemes, and argument diagramming [9]. Research which has been carried out in AAE has largely focused on the legal domain [112], with more recent work moving to online reviews[13, and online debates [14].

State of the Art. The state of the art encompasses two main approaches to automatic argumentation identification: statistical classification and rule-based parsing. In both cases, the goal is to identify and extract the parts of an argument (premises and conclusions), as well as their relationships. The work in [1] begins with experimentation on the Araucaria corpus [9], but quickly shifts focus to the annotation of a new corpus consisting of fifty-seven ECHR (European Court of Human Rights) cases. This was due to an interest in the full argumentation structure, i.e the relations between arguments, which Araucaria does not provide.

The results of this work have been outlined in Table 1. However issues with the statistical classification approach taken were also given. "This approach cannot detect the delimiters of each argument or their relations. Therefore, it is known which information forms the argumentation but not how this information is split into the different arguments." [1]. This eventually caused the research to shift towards rule-based parsing. These results are also shown in Table 1 . 
Table 1. State of the art automatic argumentation identification results (F1 Measure)

Statistical Classification Rule-base Parser

\begin{tabular}{lcc}
\hline Premise & $68.12 \%$ & $64.3 \%$ \\
Conclusion & $74.07 \%$ & $67.4 \%$ \\
Structure & N/A & $60.0 \%$ \\
\hline
\end{tabular}

\section{Contributions}

Firstly, this research will begin by providing a much needed argumentation corpus, as well as tools to enable easier production of argumentation corpora. This corpus will be annotated with tags suitable for analysis by many of the available Apache UIMA 1 Tools and components 15. Annotation is underway and a need for clear definitions has already become evident. As in previous work [12], we will attempt to establish an appropriate definition of the elementary units of argumentation. There is broad consensus that arguments are the elementary units of argumentation, but what are the elements of an argument? Initial efforts in annotating a selection of car reviews, (our Car Review Corpus - CRC) highlight the fact that sentences are not appropriate (as compared to the state of the art[1]) as the fundamental elements of an argument (i.e. a complete, conventionally punctuated sentence often cannot simply be labeled as being a single 'conclusion' or a single 'premise'). Take for example the sentence "Other weight saving measures means it is $80 \mathrm{~kg}$ lighter overall and Audi claims it is the lightest car in the class." This sentence, taken from an AA Car Review for an Audi A3, contains the conclusion, "Audi claims it is the lightest car in the class." However it also contains a premise: "Other weight saving measures means it is $80 \mathrm{~kg}$ lighter overall" which adds support to the claim. Therefore we can say that this sentence contains both a conclusion and premise. This is a comparatively simple case - a complex sentence comprising two independent clauses joined by a coordinator - and can be easily annotated. However, devising a means of identifying propositions and their role in more complex syntactic constructions will require greater effort. This research regards the proposition, classified as a conclusion or premise, as the smallest element of an argument.

Our research addresses potential issues in moving from the more structured natural language found in legal cases (state of the art), to the less structured and therefore more computationally complex domain of journalistic argumentation and consumer comment, exemplified in this case by car reviews. Our work will explore which features achieve higher accuracies given various machine learning (ML) algorithms. It will begin by using Support Vector Machines (SVMs), evaluating results against other ML algorithms such as Maximum Entropy(ME). ML has been chosen because of the positive results achieved in 11, where it was shown to obtain higher F1 measures in identifying both premises and conclusions compared to rule-based parsing.

\footnotetext{
1 http://uima.apache.org/
} 
Statistical classification, whether SVN or ME, typically encounters difficulty when faced with the challenge of identifying individual argument parts and then associating them with over-arching argumentation structures. Our research will tackle this segmentation problem by use of semantic analysis. Whilst this was explored briefly in [1, results were unfavorable. However it was stated that, "A different type of document or a more complex clustering model could achieve better results, however it was decided to leave this research line for future work."

\section{Evaluations}

The evaluation of our system's effectiveness in identifying basic units of argument will be standardised with the state of the art to enable direct comparison. The state of the art uses well known evaluation metrics to count the number of correctly classified sentences. In our work we will be counting the number of correctly classified propositions instead of sentences. Therefore, in the context of classification tasks (cf. classification between two class labels: C1 and C2) the following four terms are used to compare the given labels with the label the items actually belong to:

- True Positive $\left(T_{p}\right)$ : number of propositions correctly classified as C1

- True Negative $\left(T_{n}\right)$ : number of propositions correctly classified as C2

- False Positive $\left(F_{p}\right)$ : number of propositions incorrectly classified as C1

- False Negative $\left(F_{n}\right)$ : number of propositions incorrectly classified as C2

Precision $(\mathrm{P})$, recall( $\mathrm{R})$ and $\mathrm{F} 1$ measure are defined as follows:

$$
\mathrm{P}=\frac{T_{p}}{T_{p}+F_{p}} \quad \mathrm{R}=\frac{T_{p}}{T_{p}+F_{n}} \quad \mathrm{~F} 1=2 * \frac{P * R}{P+R}
$$

Accuracy is computed as the number of correctly classified propositions divided by the total number of propositions:

$$
\text { Accuracy }=\frac{N_{\text {corr }}}{N_{\text {total }}} \quad N_{\text {corr }}=T_{p}+T_{n} \quad N_{\text {total }}=T_{p}+T_{n}+F_{p}+F_{n}
$$

\section{Work Plan}

The initial effort has been on the creation of tools to help annotate a new argumentative corpus, the Car Reviews Corpus (CRC). These tools, based upon UIMA [15, can be used to annotate text. The annotations created are then capable of being utilised by any new or existing UIMA components.

The next stage of work is the annotation of propositions within the car review texts by several human annotators, followed by a study of annotator agreement. This will lead to the task of training a classifier to automatically identify propositions in unseen text in the same domain. 
Upon successful completion, attention will shift back to fully annotating the CRC with argumentative annotations, describing the argument parts and structure. Once again, an evaluation of annotator agreement will be carried out before proceeding to the task of classifying argumentative propositions from nonargumentative, followed by classification of conclusions and premises.

The final stage of this work will be the investigation into a complex semantic clustering model to enable the automatic construction of an argument tree from an unseen text in a domain similar to that of the training corpus.

Acknowledgements. This work is supported by SAP AG and the Invest NI Collaborative Grant for R\&D - RD1208002.

Supervisors - Ian O'Neill \& Jun Hong (School of Electronics, Electrical Engineering and Computer Science, Queen's University Belfast, Belfast, BT7 1NN)

\section{References}

1. Mochales Palau, R.: Automatic Detection and Classification of Argumentation in a Legal Case. PhD thesis, KU Leuven (2011)

2. Walton, D.: Argument mining by applying argumentation schemes. Studies in Logic 4(1), 38-64 (2011)

3. Besnard, P., Hunter, A.: Elements of argumentation, vol. 47. MIT Press (2008)

4. Walton, D.: Argumentation Theory: A Very Short Introduction. In: Rahwan, I., Simari, G.R. (eds.) Argumentation in Artificial Intelligence, 1st edn., pp. 1-22. Springer, US (2009)

5. Baker, G.P., Huntington, H.B.: The Principles of Argumentation. Ginn \& Company (1905)

6. Govier, T.: Critical thinking as argument analysis? Argumentation 3(2), 115-126 (1989)

7. Copi, I., Cohen, C.: Introduction to Logic, 11th edn. Pearson Eductaion (2001)

8. Blair, J.A., Tindale, C.W.: Groundwork in the Theory of Argumentation. Argumentation Library, vol. 21. Springer (2012)

9. Reed, C.: Araucaria: Software for argument analysis, diagramming and representation. International Journal of AI Tools 13(4), 961-980 (2004)

10. Teufel, S.: Argumentative Zoning: Information Extraction from Scientific Text University of Edinburgh. PhD thesis (1999)

11. Mann, W.C., Thompson, S.A.: Rhetorical structure theory: A framework for the analysis of texts. IPRA Papers in Pragmatics 1(1), 79-105 (1987)

12. Mochales Palau, R., Moens, M.F.: Argumentation Mining: The Detection, Classification and Structure of Arguments in Text. In: Proceedings of the 12th International Conference on Artificial Intelligence and Law, pp. 98-107. ACM (2009)

13. Schneider, J., Wyner, A.: Identifying Consumers Arguments in Text. In: SWAIE 2012: Semantic Web and Information Extraction (2012)

14. Cabrio, E., Villata, S.: Natural Language Arguments: A Combined Approach. In: 20th European Conference on Artificial Intelligence, ECAI 2012 (2012)

15. Ferrucci, D., Lally, A.: UIMA: An Architectural Approach to Unstructured Information Processing in the Corporate Research Environment. Natural Language Engineering 10(3-4), 327-348 (2004) 